\title{
La religión de los estudiantes y la incidencia en su formación profesional, ULEAM-2018
}

The religion of the students and the incidence in their professional formation, ULEAM-2018

\section{A religião dos estudantes e a incidência em sua formação profissional, ULEAM- 2018}

Patricia Josefa López-Mero ${ }^{\mathrm{I}}$

pattylopez@hotmail.com

Katherine Monserrate Cevallos-Alcívar II

katherinecevallos@gmail.com

Recibido: 19 de abril de 2019 *Aceptado: 18 de junio de 2019* Publicado: 05 de julio de 2019

1. Magister en Gerencia Educativa, Diploma Superior en Intervención Social, Licenciada En Ciencias de la Educación Especialidad Castellano y Literatura, Profesora de Segunda Enseñanza Especialidad Castellano y Literatura, Licenciada en Trabajo Social Especialidad Atención a la Familia y al Desarrollo Comunitario, Docente de la Universidad Laica Eloy Alfaro de Manabí, Manta, Ecuador.

II. Licenciada en Trabajo Social, Egresada de la Facultad de Trabajo Social de la Universidad Laica Eloy Alfaro de Manabí, Manta, Ecuador. 


\title{
Resumen
}

El objetivo principal del presente artículo es determinar como la religión incide en el proceso de enseñanza aprendizaje como parte de la formación profesional de los estudiantes de la carrera de Trabajo Social. Los fundamentos teóricos que sustentan la investigación, se reflejan en las variables identificadas, la conceptualización sobre la religión, tipos de religión, influencia de la religión en la educación superior. Por otra parte, las conceptualizaciones de la formación profesional, Trabajo Social y cuáles son sus roles, funciones, además se hace referencia a los modelos de intervención de Trabajo Social como: psicosocial, centrado en la tarea, modelo de intervención en crisis, ecológica y sistémica.

La metodología tributa a una investigación de tipo cuantitativa, de campo y bibliográfica; se enfocó en determinar la incidencia de la religión en la formación profesional; se aplicaron encuestas a 107 estudiantes de los niveles y paralelos; se utilizó los métodos deductivo, inductivo, descriptivo y estadístico; la misma que determinó las siguientes conclusiones: Los estudiantes de cuarto "A", octavo "A"-"B", noveno "A" de la facultad de Trabajo Social profesan las siguientes religiones: El 77,57\% es Católico; el 7,48\% Cristiano; el 2,80\% Evangélico; el 2,80\% Ateo; el 1,87\% mormón y el 7,48\% otras religiones. Los estudiantes encuestados consideran que la religión influye en los ámbitos: Familiar el 52\%, Personal 31\%, Social 8\%, otros ámbitos el 7\% y Educativo 2\%; Para los estudiantes encuestados de la Facultad de Trabajo Social, la religión no influye en su formación profesional.

Palabras clave: la religión; formación profesional; enseñanza; aprendizaje; estudiantes; trabajo social.

\begin{abstract}
The main objective of this article is to determine how religion affects the teaching-learning process as part of the professional training of students of the Social Work career. The theoretical foundations that sustain the research are reflected in the identified variables, the conceptualization of religion, types of religion, the influence of religion in higher education. On the other hand, the conceptualizations of vocational training, Social Work and what their roles, functions, are also
\end{abstract}


referred to Social Work intervention models as: psychosocial, task-centered, crisis intervention model, ecological and systemic

The methodology is subject to quantitative, field and bibliographic research; focused on determining the incidence of religion in vocational training; surveys were applied to 107 students of the levels and parallels; the deductive, inductive, descriptive and statistical methods were used; the same that determined the following conclusions: The students of room "A", eighth "A" - "B", ninth "A" of the faculty of Social Work profess the following religions: $77.57 \%$ is Catholic; $7.48 \%$ Christian; 2.80\% Evangelical; 2.80\% Atheist; 1.87\% Mormon and 7.48\% other religions. The students surveyed consider that religion influences the following areas: Family 52\%, Personal 31\%, Social 8\%, Other areas 7\% and Educational 2\%; For the students surveyed of the Faculty of Social Work, religion does not influence their professional training.

Key words: the religion; vocational training; teaching; learning; students; social work.

\section{Resumo}

O objetivo principal deste artigo é determinar como a religião afeta o processo ensinoaprendizagem como parte da formação profissional de estudantes da carreira do Serviço Social. Os fundamentos teóricos que sustentam a pesquisa refletem-se nas variáveis identificadas, na conceituação da religião, nos tipos de religião, na influência da religião no ensino superior. Por outro lado, as conceituações de formação profissional, Serviço Social e quais os seus papéis, funções, são também referidos aos modelos de intervenção do Serviço Social como: psicossocial, centrado na tarefa, modelo de intervenção em crises, ecológico e sistêmico

A metodologia está sujeita a pesquisas quantitativas, de campo e bibliográficas; concentrou-se em determinar a incidência da religião na formação profissional; inquéritos foram aplicados a 107 estudantes dos níveis e paralelos; os métodos dedutivo, indutivo, descritivo e estatístico foram utilizados; o mesmo que determinou as seguintes conclusões: Os alunos da sala "A", oitavo "A" "B", nono "A" da faculdade de Serviço Social professam as seguintes religiões: 77,57\% é católica; 7,48\% cristão; $2,80 \%$ evangélico; 2,80\% ateu; 1,87\% Mórmon e 7,48\% outras religiões. Os estudantes pesquisados consideram que a religião influencia nas seguintes áreas: Família 52\%, Pessoal 31\%, Social 8\%, Outras áreas 7\% e Educacional 2\%; Para os alunos pesquisados da Faculdade de Serviço Social, a religião não influencia sua formação profissional. 
Palavras-chave: a religião; formação profissional; ensino; aprendizagem estudantes; trabalho social.

\section{Introducción}

El presente artículo surge a raíz del conocimiento de la manera en que algunos profesionales en la actualidad utilizan la intervención para encaminar a las personas hacia la religión. Se evidencia en algunas investigaciones que la religión es aprovechada para que de forma consciente se pueda conectar y relacionarse con la persona, la manera de utilizar el entorno y la naturaleza para generar paz, amabilidad y cooperación con el mundo, provocando muchas veces un cambio de actitudes en las personas, ya que muchas no profesan ninguna clase de religión.

Lo anteriormente expuesto, fue la razón que motivo la realización de este artículo, cuya finalidad es conocer cuáles son las causas y efectos de esta problemática. Al utilizar la religión dentro de la intervención se podría afirmar que se pierde la línea con la que la carrera forma a los profesionales en las cuales debe utilizar técnicas y metodologías para poder llevar a cabo una buena intervención. La formulación del problema de la investigación se sustenta bajo la siguiente interrogante ¿La religión de los estudiantes de la carrera de Trabajo Social incide en la formación profesionalULEAM 2018? En cuanto a los objetivos que se plantean en la investigación se pueden mencionar como Objetivo general: Determinar la incidencia de la religión de los estudiantes de la carrera de Trabajo Social en la formación profesional-ULEAM 2018 y los Objetivos específicos:

- Conocer los tipos de religiones que profesan los estudiantes de la Facultad de Trabajo Social.

- Identificar la aplicación de la religión de los estudiantes en las aulas de clases de la carrera de Trabajo Social.

- Determinar de qué manera incide la religión de los estudiantes en su formación profesional.

Las HIPÓTESIS que fundamentan la presente investigación: La religión de los estudiantes de la carrera de Trabajo Social incide negativamente en su formación profesional-Uleam 2018. La Variable dependiente: La formación profesional y la Variable independiente: La religión. 
Los temas relacionados a la religión deben ser considerados en los ámbitos Familiar, Personal, Social y en la educación; en el proceso de enseñanza-aprendizaje no se recomienda la inclusión de temas religiosos para la solución de una problemática social y que, a la hora de elegir una carrera, sea por vocación y convicción y no un espacio para practicar la religión.

\section{Metodología}

El presente artículo se basa en una metodología cuantitativa que busca evaluar cómo la religión de los estudiantes incide en su formación profesional en la Facultad de Trabajo Social de la universidad Laica "Eloy Alfaro" de Manabí. Se utilizó la investigación de la bibliografía para recopilar información, teorías de diferentes investigaciones respecto al tema de la religión y como incide en la formación profesional de los estudiantes desde el enfoque de Trabajo Social. Es una investigación de campo por lo que se acudió a las aulas de los diferentes paralelos seleccionados para aplicar las encuestas a los estudiantes y así poder obtener los resultados y poder determinar sí la religión incide en la formación profesional de los estudiantes en la Facultad de Trabajo Social.

La investigación es exploratoria debido al acercamiento que se realizó para conocer de manera general si existe influencia de la religión en la formación profesional de los estudiantes y es descriptiva porque se realizó una descripción de las características de la población o fenómeno estudiado.

La población objeto de estudio fue 569 estudiantes de la Facultad de Trabajo Social de la Universidad Laica "Eloy Alfaro” de Manabí del cantón Manta. Asimismo, la muestra seleccionada fue 107 estudiantes para conocer cuáles son los diferentes tipos de empleada fue la recolección de datos por medio de encuestas a los estudiantes de cuarto "A", octavo "A"-“B” y noveno "A", está técnica ayudó de forma individual, conocer las diferentes opiniones e incidencias de la religión en los estudiantes en la formación profesional.

Para realizar este tipo de encuestas fue necesario lograr una adecuada interacción con los estudiantes, siendo este el primer contacto, lo que permitió conocer el lugar y número de estudiantes con los que se trabajaría.

Los Recursos Humanos que permitieron llevar a cabo la presente investigación fueron: 
- $\quad$ Investigadora

- $\quad$ Tutora

- $\quad$ Estudiantes

Los Recursos Materiales utilizados: Resma de papel, Laptop, Esferográficos, Impresora, Cd's, Lápices, Cuaderno de notas

Dentro de los Recursos Económicos se puede mencionar: Movilización, Viáticos, Alimentación, Impresiones

\section{Resultados y Discusión}

\section{Análisis e Interpretación de Resultados}

Tabla 1 ¿Qué tipo de religión usted profesa?

\begin{tabular}{|l|l|l|}
\hline OPCIONES & TOTAL DE RESPUESTAS & PORCENTAJE \\
\hline Católica & 83 & $77,57 \%$ \\
\hline Evangélica & 3 & $2,80 \%$ \\
\hline Testigo de Jehová & 0 & $0,00 \%$ \\
\hline Cristiano & 8 & $7,48 \%$ \\
\hline Mormón & 2 & $1,87 \%$ \\
\hline Ateo & 3 & $2,80 \%$ \\
\hline Otros & 8 & $7,48 \%$ \\
\hline TOTAL & 107 & $100,00 \%$ \\
\hline
\end{tabular}

FUENTE: Estudiantes de los niveles cuarto "A", octavo "A" $y$ "B" y noveno "A" de la facultad de Trabajo Social 
Elaborado por: Katherine Monserrate Cevallos Alcívar

\section{PORCENTAJE}

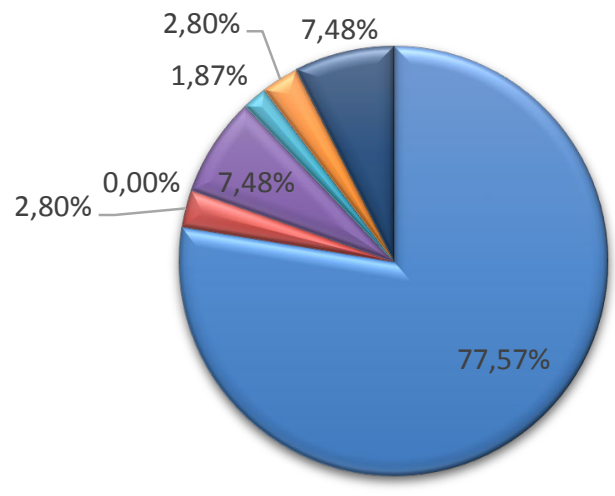

\Catolica $\square$ Evagelica $\square$ Testigo de Jehova $\square$ Cristiano $\square$ Mormón $\square$ Ateo $\square$ Otros

Gráfico 1 Qué tipo de religión usted profesa

FUENTE: Estudiantes de los niveles cuarto "A", octavo "A"

ELABORADO POR: Katherine Monserrate Cevallos Alcívar.

Interpretación. - Se puede observar en el gráfico que el 77,57\% de los estudiantes de la facultad de Trabajo Social profesan la religión católica, mientras que el 7,48 \% la religión cristiana y otro tipo de religión; además un $2,80 \%$ de la población encuestada refleja que es evangélica y el otro porcentaje es atea, por lo que considerablemente el mayor porcentaje lo adquiere la religión católica.

Independientemente del hecho que seas practicante o no, te consideras una persona:

Tabla 2 Independientemente del hecho que seas practicante o no, te consideras una persona:

\begin{tabular}{|l|l|l|}
\hline OPCIÓN & TOTAL DE RESPUESTAS & PORCENTAJE \\
\hline MUY RELIGIOSO & 11 & $10,28 \%$ \\
\hline RELIGIOSO & 53 & $49,53 \%$ \\
\hline POCO RELIGIOSO & 31 & $28,97 \%$ \\
\hline NADA RELIGIOSO & 12 & $11,21 \%$ \\
\hline
\end{tabular}




\begin{tabular}{|l|l|l|}
\hline TOTAL & 107 & $100,00 \%$ \\
\hline
\end{tabular}

FUENTE: Estudiantes de los niveles cuarto "A", octavo "A" y "B" y noveno "A" de la facultad de Trabajo Social.

ELABORADO POR: Katherine Monserrate Cevallos Alcíva

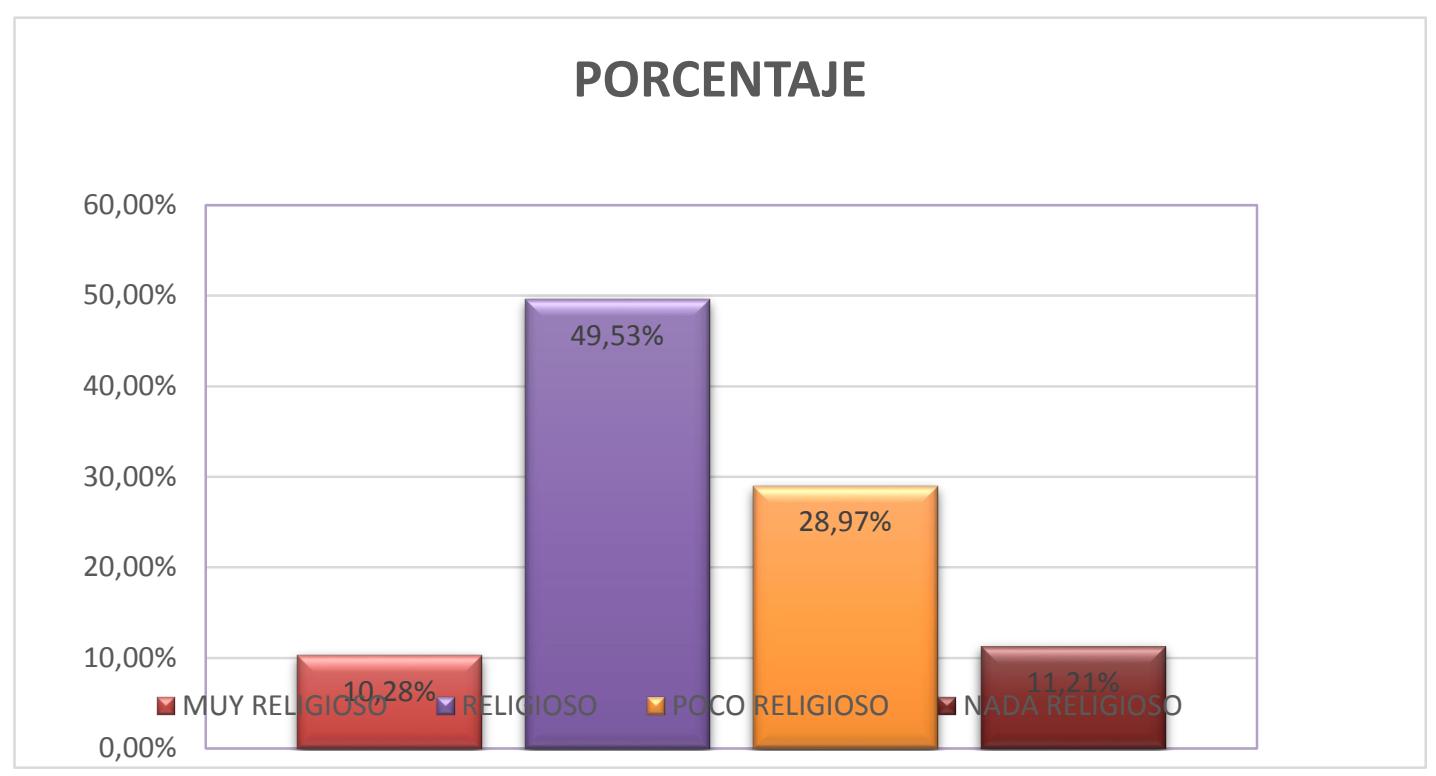

Gráfico 2 Independientemente del hecho que seas practicante o no, te consideras una persona:

FUENTE: Estudiantes de los niveles cuarto "A", octavo "A" y "B" y noveno "A" de la facultad de Trabajo Social.

ELABORADO POR: Katherine Monserrate Cevallos Alcívar.

Interpretación. - El resultado muestra que el 49,53\% de los estudiantes encuestados se consideran religiosos, por otra parte el $28,97 \%$ de los mismos se consideran poco religiosos; el 11,21\% son nada religioso y finalmente con un porcentaje del 10,28\% los estudiantes se consideran muy religiosos

¿En qué ámbitos de tu vida influye más tu religión, o tus creencias religiosas?

Tabla 3 ¿En qué ámbitos de tu vida influye más tu religión, o tus creencias religiosas?

\begin{tabular}{|l|l|l|}
\hline ÁMBITOS & TOTAL DE RESPUESTAS & PORCENTAJE \\
\hline PERSONAL & 33 & $30,84 \%$ \\
\hline LABORAL & 0 & $0,00 \%$ \\
\hline
\end{tabular}




\begin{tabular}{|l|l|l|} 
SOCIAL & 8 & $7,48 \%$ \\
\hline FAMILIAR & 56 & $52,34 \%$ \\
\hline EDUCATIVO & 2 & $1,87 \%$ \\
\hline OTROS & 8 & $7,48 \%$ \\
\hline TOTAL & 107 & $100,00 \%$ \\
\hline
\end{tabular}

FUENTE: Estudiantes de los niveles cuarto "A", octavo "A" y "B" y noveno "A" de la facultad de Trabajo Social.

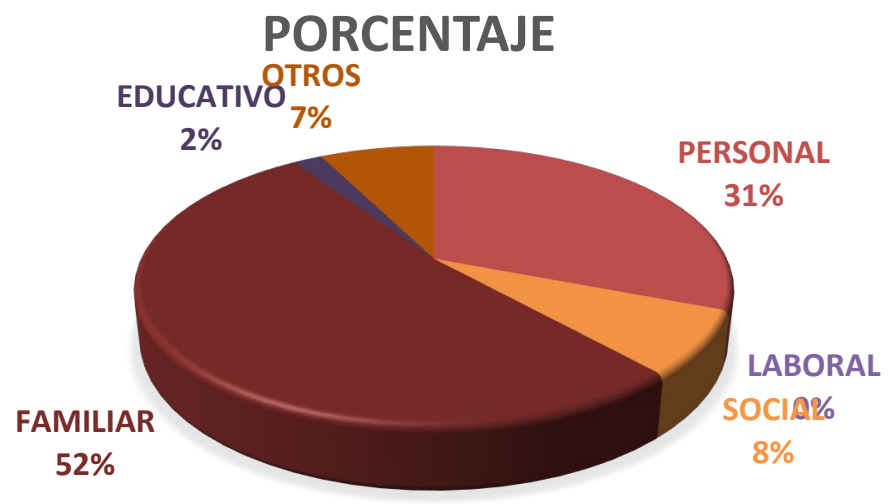

GPERSONAL

口LABORAL ISOCIAL

FAMILIAR

EDUCATIVO

GOTROS

Gráfico 3 ¿En qué ámbitos de tu vida influye más tu religión, o tus creencias religiosas?

\section{ELABORADO POR: Katherine Monserrate Cevallos Alcívar}

Fuente: Estudiantes de los niveles cuarto "A", octavo "A" $y$ "B" y noveno "A" de la facultad de Trabajo Social.

Interpretación. - Se puede evidenciar que el 52\% de los estudiantes encuestados consideran que uno de los ámbitos que más influye la religión es a nivel familiar, por otra parte, el 31\% considera que esta influye a nivel personal; el 8\% de estudiantes manifestaron que influye en la parte social; el $2 \%$ de la población creen que la religión de una u otra manera influye en el ámbito educativo y finalmente con el 7\% unos indican que existen otros ámbitos que influye la religión. 


\section{¿La religión forma parte de su formación profesional?}

Tabla 4 ¿La religión forma parte de su formación profesional?

\begin{tabular}{|l|l|l|}
\hline OPCIÓN & TOTAL DE RESPUESTAS & PORCENTAJE \\
\hline SI & 29 & $27,10 \%$ \\
\hline NO & 78 & $72,90 \%$ \\
\hline TOTAL & 107 & $100,00 \%$ \\
\hline
\end{tabular}

FUENTE: Estudiantes de los niveles cuarto “A”, octavo "A" y "B" y noveno "A" de la facultad de Trabajo Social.

ELABORADO POR: Katherine Monserrate Cevallos Alcívar.

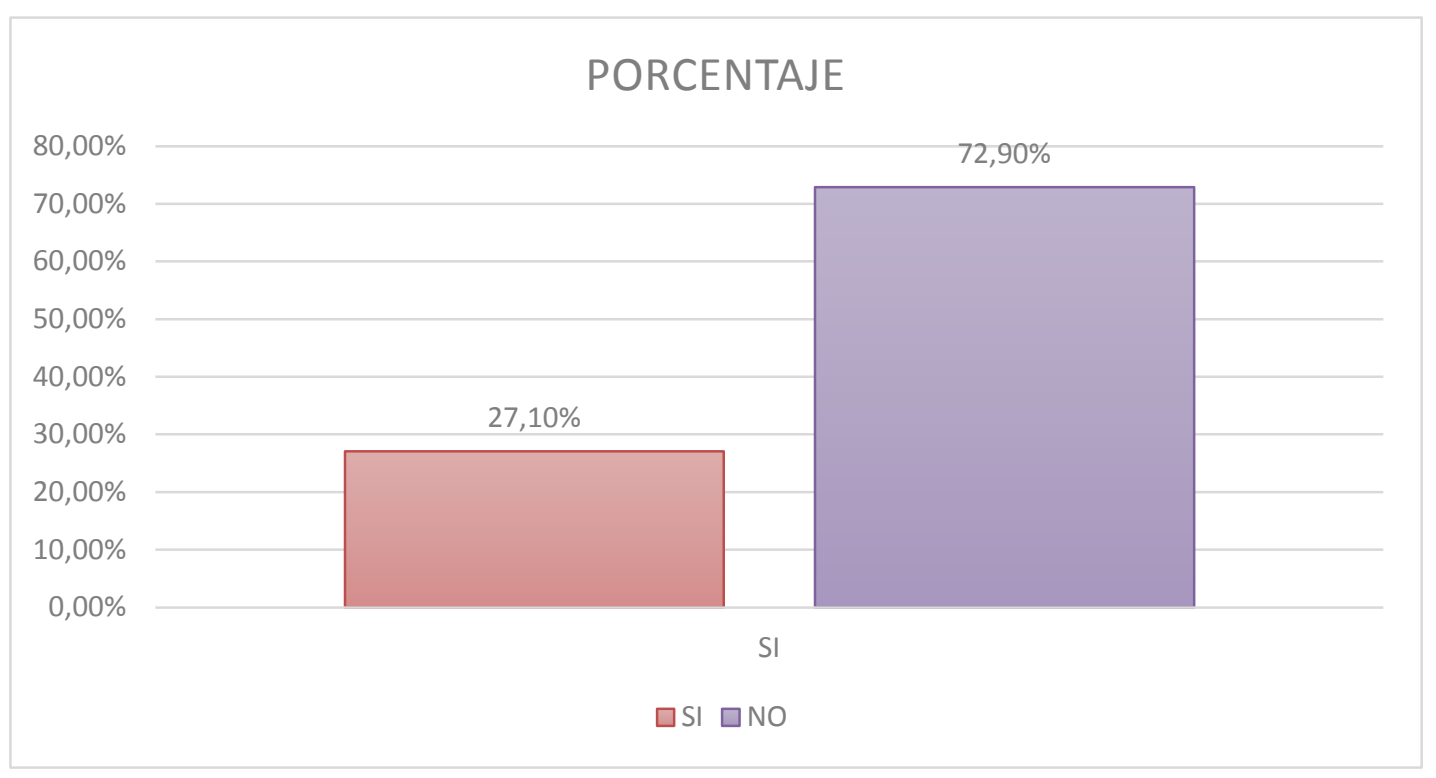

Gráfico 4 ¿La religión forma parte de su formación profesional?

FUENTE: Estudiantes de los niveles cuarto "A", octavo "A" y "B" y noveno "A" de la facultad de Trabajo Social.

ELABORADO POR: Katherine Monserrate Cevallos Alcívar.

Interpretación. - Se puede evidenciar claramente que el 72,90\% de la población estudiantil encuestada considera que la religión no forma parte de la formación profesional; mientras tanto el 27,10\% considera que de alguna u otra manera la religión forma parte de su formación profesional. 
¿La religión estaría influenciada por una serie de factores que se relacionan dialécticamente con una sociedad y una cultura?

Tabla 5 ¿La religión estaría influenciada por una serie de factores que se relacionan dialécticamente con una sociedad y una cultura?

\begin{tabular}{|l|l|l|}
\hline OPCIÓN & TOTAL DE RESPUESTAS & PORCENTAJE \\
\hline SIEMPRE & 9 & $8,41 \%$ \\
\hline A VECES & 55 & $51,40 \%$ \\
\hline FRECUENTEMENTE & 27 & $25,23 \%$ \\
\hline NUNCA & 16 & $14,95 \%$ \\
\hline TOTAL & 107 & $100,00 \%$ \\
\hline
\end{tabular}

FUENTE: Estudiantes de los niveles cuarto "A", octavo "A" $\mathrm{y}$ "B" y noveno "A" de la facultad de Trabajo Social.

ELABORADO POR: Katherine Monserrate Cevallos Alcívar.

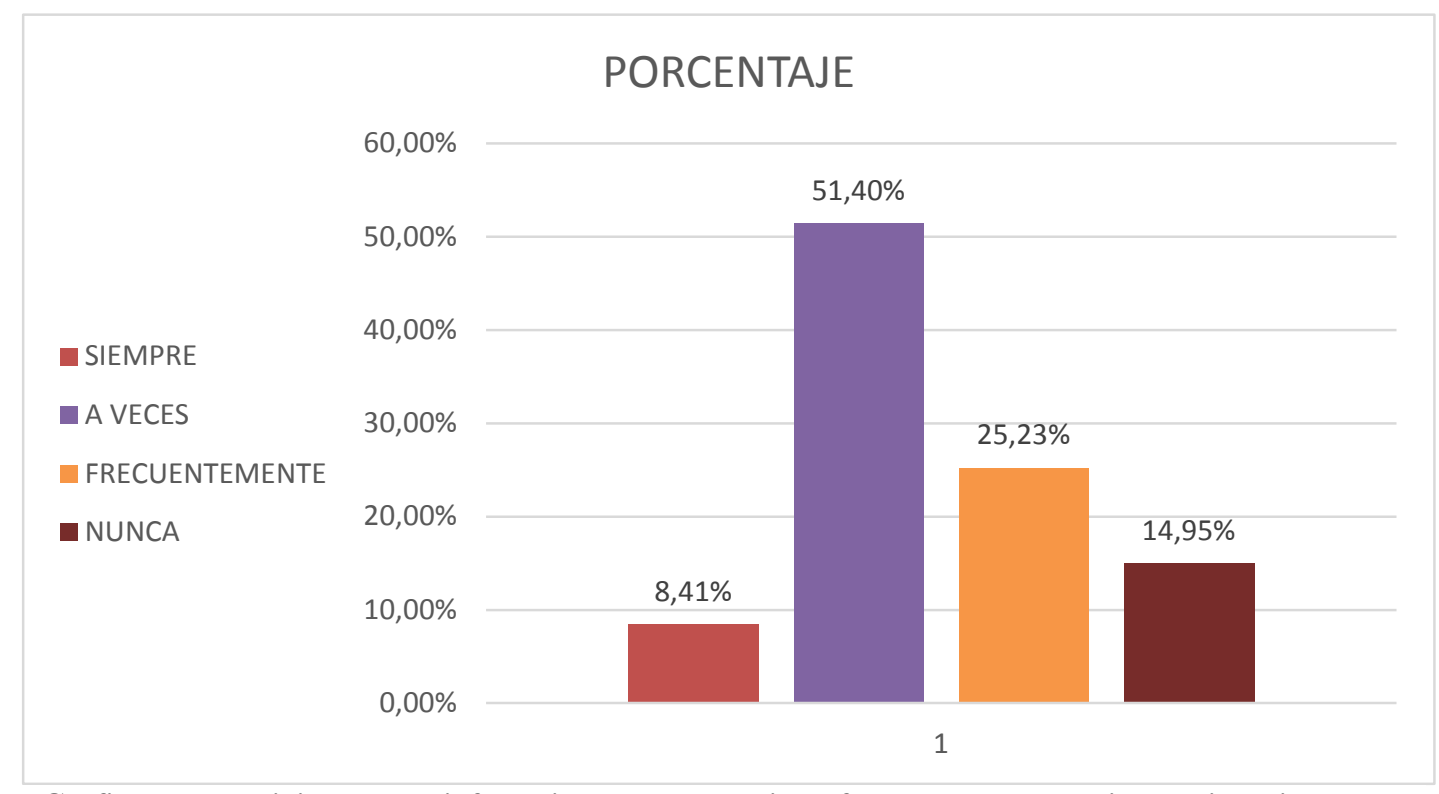

Gráfico 5 ¿La religión estaría influenciada por una serie de factores que se relacionan dialécticamente con una sociedad y una cultura?

Fuente: Estudiantes de los niveles cuarto "A", octavo "A" y "B" y noveno "A" de la facultad de Trabajo Social. Elaborado por: Katherine Monserrate Cevallos Alcívar. 
Interpretación.- Mediante el cuadro estadístico se puede observar que el 51,40\% de los estudiantes encuestados piensan que la religión si estaría influenciada por la sociedad y la cultura, mientras que el 25,23\% piensa que frecuentemente estos factores influyen considerablemente, por otra parte el $14,95 \%$ considera que estos factores no influyen de ninguna manera y finalmente con el $8,41 \%$ la población estudiantil a través de las encuestas manifiesta que siempre la cultura y la sociedad influyen dialécticamente con la religión.

¿El desarrollo de la religión es favorable en los procesos de la formación profesional?

Tabla 6 ¿El desarrollo de la religión es favorable en los procesos de la formación profesional?

\begin{tabular}{|l|l|l|}
\hline OPCIÓN & TOTAL DE RESPUESTAS & PORCENTAJE \\
\hline SIEMPRE & 3 & $2,80 \%$ \\
\hline A VECES & 67 & $62,62 \%$ \\
\hline NUNCA & 37 & $34,58 \%$ \\
\hline TOTAL & 107 & $100,00 \%$ \\
\hline
\end{tabular}

FUENTE: Estudiantes de los niveles cuarto "A", octavo "A" $y$ "B" y noveno "A" de la facultad de Trabajo Social.

ELABORADO POR: Katherine Monserrate Cevallos Alcívar.

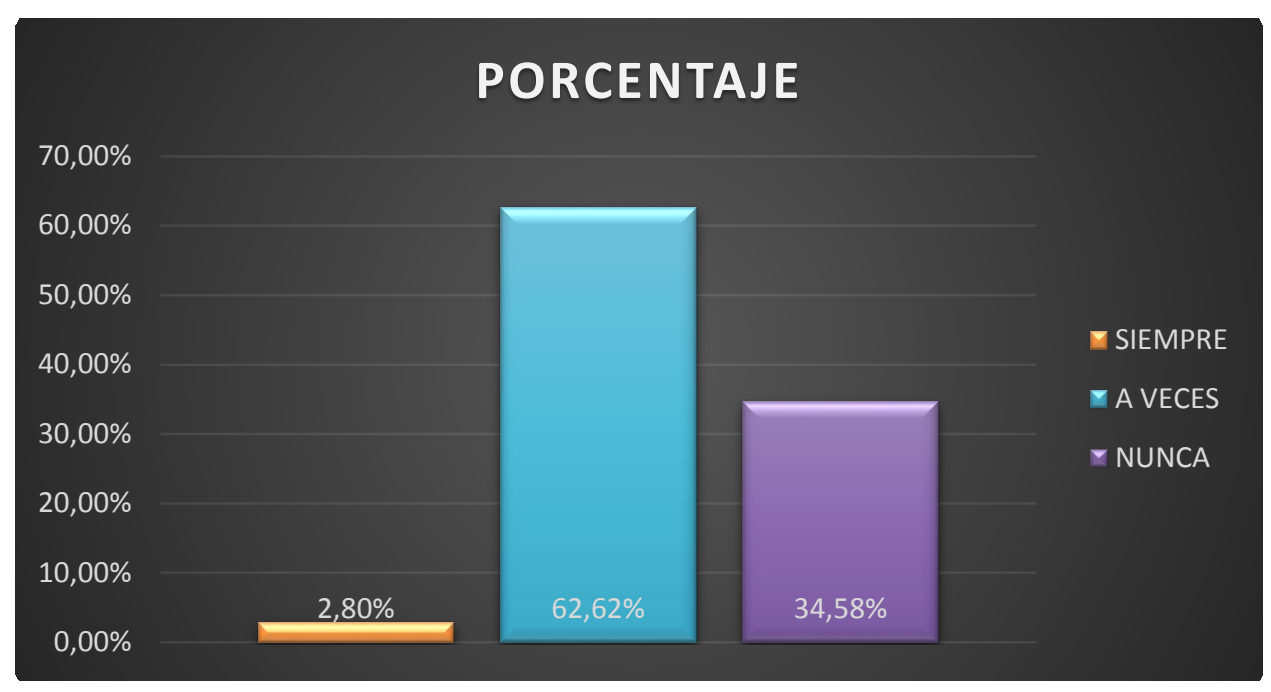

Gráfico 6 ¿El desarrollo de la religión es favorable en los procesos de la formación profesional?

FUENTE: Estudiantes de los niveles cuarto “A", octavo "A" y "B" y noveno "A" de la facultad de Trabajo Social. ELABORADO POR: Katherine Monserrate Cevallos Alcívar. 
Interpretación.- Se puede evidenciar que el $62,62 \%$ de los estudiantes encuestados consideran que a veces es favorable el desarrollo de la religión en sus procesos de formación profesional, mientras que por otra parte el 34,58\% cree que nunca se debería de inmiscuir la religión en el proceso de formación, ya que dentro de la carrera se trabaja a través de metodologías, métodos e instrumentos y modelos de intervención profesional; el 2,80\% de los estudiantes manifestaron que para ellos siempre deben ser favorable el desarrollo de la religión a lo largo de su formación.

\section{¿La religión tiene que ver con las características personales y profesionales las cuales vamos} desarrollando en el aula de clases?

Tabla 7 ¿La religión tiene que ver con las características personales y profesionales las cuales vamos desarrollando en el aula de clases?

\begin{tabular}{|l|l|l|}
\hline OPCIÓN & TOTAL DE RESPUESTAS & PORCENTAJE \\
\hline SIEMPRE & 2 & $1,87 \%$ \\
\hline A VECES & 55 & $51,40 \%$ \\
\hline NUNCA & 50 & $46,73 \%$ \\
\hline TOTAL & 107 & $100,00 \%$ \\
\hline
\end{tabular}

FUENTE: Estudiantes de los niveles cuarto "A", octavo "A" y "B" y noveno "A" de la facultad de Trabajo Social

ELABORADO POR: Katherine Monserrate Cevallos Alcívar

\section{PORCENTAJE}

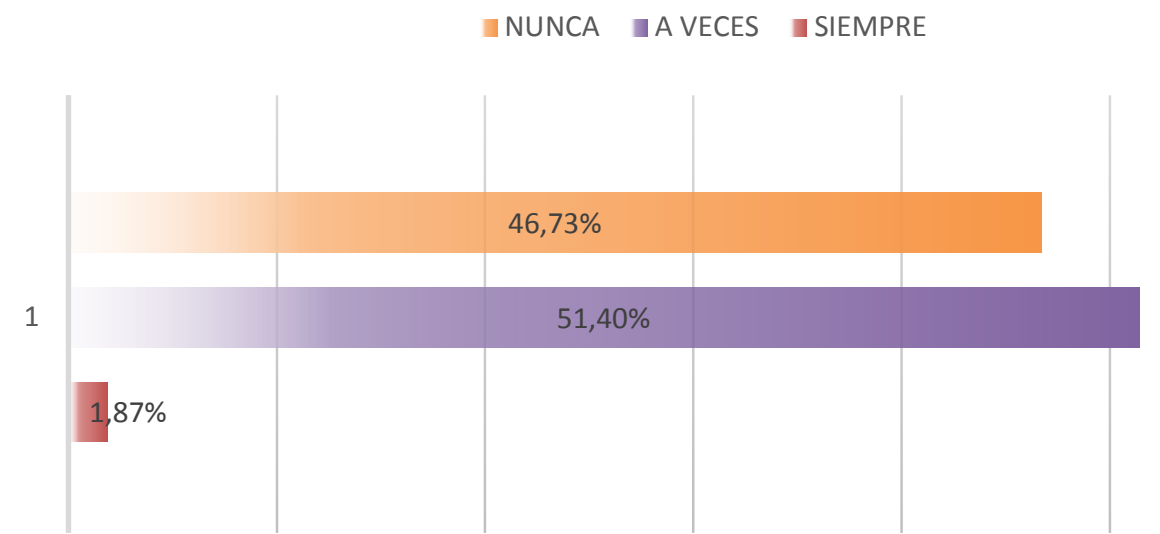

Gráfico 7 ¿La religión tiene que ver con las características personales y profesionales las cuales vamos desarrollando en el aula de clases? 
FUENTE: Estudiantes de los niveles cuarto "A", octavo "A" $y$ "B" y noveno "A" de la facultad de Trabajo Social ELABORADO POR: Katherine Monserrate Cevallos Alcívar

Interpretación.- A través de cuadro estadístico se puede evidenciar que el $51,40 \%$ de los estudiantes de la carrera creen que a veces la religión tiene que ver con sus características personales y profesionales las cuales se desarrollan en las aulas de clases, por otro lado con un porcentaje de 46,73 los estudiantes manifiestan que no es necesaria la religión para poder desarrollarse de manera correcta en el aula de clases, finalmente con un bajo porcentaje de 1,87 manifestaron que para ellos siempre es importante que su religión esté inmiscuida a la hora de sus clases y al desarrollarse a lo largo de su carrera profesional.

¿La religión articulada con sus necesidades personales, le confiere al creyente un instrumento indispensable para enfrentar problemas al momento de la formación profesional?

Tabla 8 ¿La religión articulada con sus necesidades personales, le confiere al creyente un instrumento indispensable para enfrentar problemas al momento de la formación profesional?

\begin{tabular}{|l|l|l|}
\hline OPCIÓN & TOTAL DE RESPUESTAS & PORCENTAJE \\
\hline SIEMPRE & 4 & $3,74 \%$ \\
\hline A VECES & 47 & $43,93 \%$ \\
\hline FRECUENTEMENTE & 20 & $18,69 \%$ \\
\hline NUNCA & 36 & $33,64 \%$ \\
\hline TOTAL & 107 & $100,00 \%$ \\
\hline
\end{tabular}

Fuente: Estudiantes de los niveles cuarto "A", octavo "A" y "B" y noveno "A" de la facultad de Trabajo Social. Elaborado por: Katherine Monserrate Cevallos Alcívar. 


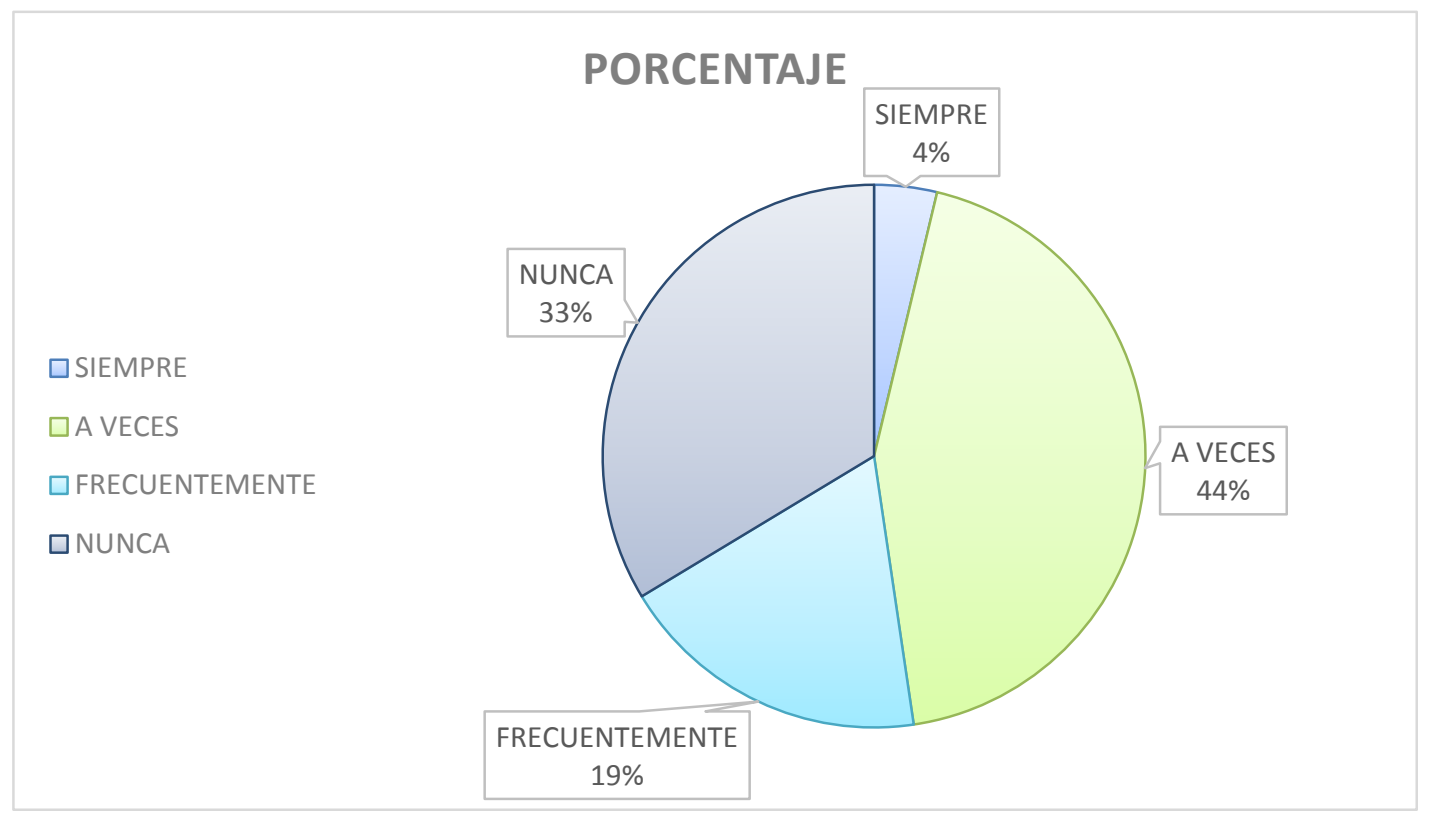

Gráfico 8 ¿La religión articulada con sus necesidades personales, le confiere al creyente un instrumento indispensable para enfrentar problemas al momento de la formación profesional?

Fuente: Estudiantes de los niveles cuarto "A", octavo "A" y "B" y noveno "A" de la facultad de Trabajo Social Elaborado por: Katherine Monserrate Cevallos Alcívar

Interpretación.- Se evidencia que el 44\% de los estudiantes a los cuales se les realizo la encuesta consideran que a veces la religión se articula con sus necesidades y que esto les ayuda como instrumento a la hora de enfrentar las problemáticas que se vayan presentando a lo largo de la formación profesional, con el 33\% los estudiantes de la carrera piensan que no es necesario recurrir a la religión porque la parte académica es lo realmente importante para tener una formación profesional de éxito, con el 19\% consideran que con frecuencia la religión es un instrumento que ayudará a enfrentar los problemas que se le presente, y finalmente como se puede observar en el cuadro estadístico el 4\% de los estudiantes creen que para ellos siempre es importante tener presente la religión, ya sea para su formación profesional o para su diario vivir.

\section{¿La religión deja de ser un elemento complementario de la realidad para convertirse en una instancia imprescindible de la vida social?}


Tabla 9 ¿La religión deja de ser un elemento complementario de la realidad para convertirse en una instancia imprescindible de la vida social?

\begin{tabular}{|l|l|l|}
\hline OPCIÓN & TOTAL DE RESPUESTAS & PORCENTAJE \\
\hline SIEMPRE & 8 & $7,48 \%$ \\
\hline CON MUCHA FRECUENCIA & 57 & $53,27 \%$ \\
\hline NUNCA & 42 & $39,25 \%$ \\
\hline TOTAL & 107 & $100,00 \%$ \\
\hline
\end{tabular}

Fuente: Estudiantes de los niveles cuarto "A", octavo "A" y "B" y noveno "A" de la facultad de Trabajo Social. Elaborado por: Katherine Monserrate Cevallos Alcívar.

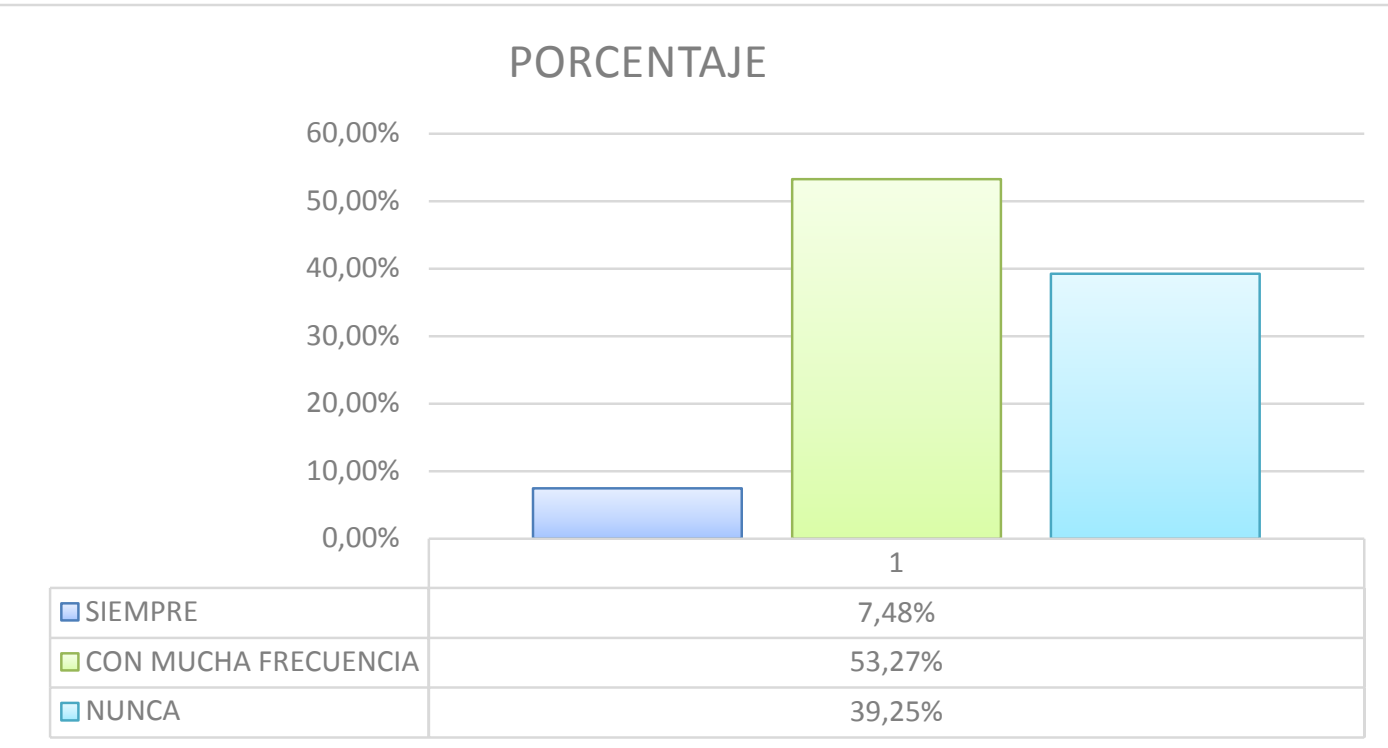

Gráfico 9 ¿La religión deja de ser un elemento complementario de la realidad para convertirse en una instancia imprescindible de la vida social?

Fuente: Estudiantes de los niveles cuarto "A", octavo "A" y "B" y noveno "A" de la facultad de Trabajo Social. Elaborado por: Katherine Monserrate Cevallos Alcívar

Interpretación. - Se puede evidenciar que el 53,27\% de los encuestados consideran que con mucha frecuencia la religión se convierte en algo indispensable para su vida social, mientras que el 39,25\% cree que la religión nunca debería de convertirse en algo indispensable sino más bien saber vivir 
con ella y con el 7,48\% la población estudiantil a la cual se encuestó, considera que para ellos la religión siempre debe de estar primero en cualquier ámbito en el que se desenvuelvan.

\section{¿Considera usted que dentro del proceso de enseñanza y aprendizaje se incluya cualquier} tipo de religión para la solución de una problemática de intervención profesional?

Tabla 10 ¿Considera usted que dentro del proceso de enseñanza y aprendizaje se incluya cualquier tipo de religión para la solución de una problemática de intervención profesional?

\begin{tabular}{|l|l|l|}
\hline OPCIÓN & TOTAL DE RESPUESTAS & PORCENTAJE \\
\hline SI & 22 & $20,56 \%$ \\
\hline NO & 85 & $79,44 \%$ \\
\hline TOTAL & 107 & $100,00 \%$ \\
\hline
\end{tabular}

Fuente: Estudiantes de los niveles cuarto "A", octavo "A" $y$ "B" y noveno "A" de la facultad de Trabajo Social Elaborado por: Katherine Monserrate Cevallos Alcívar

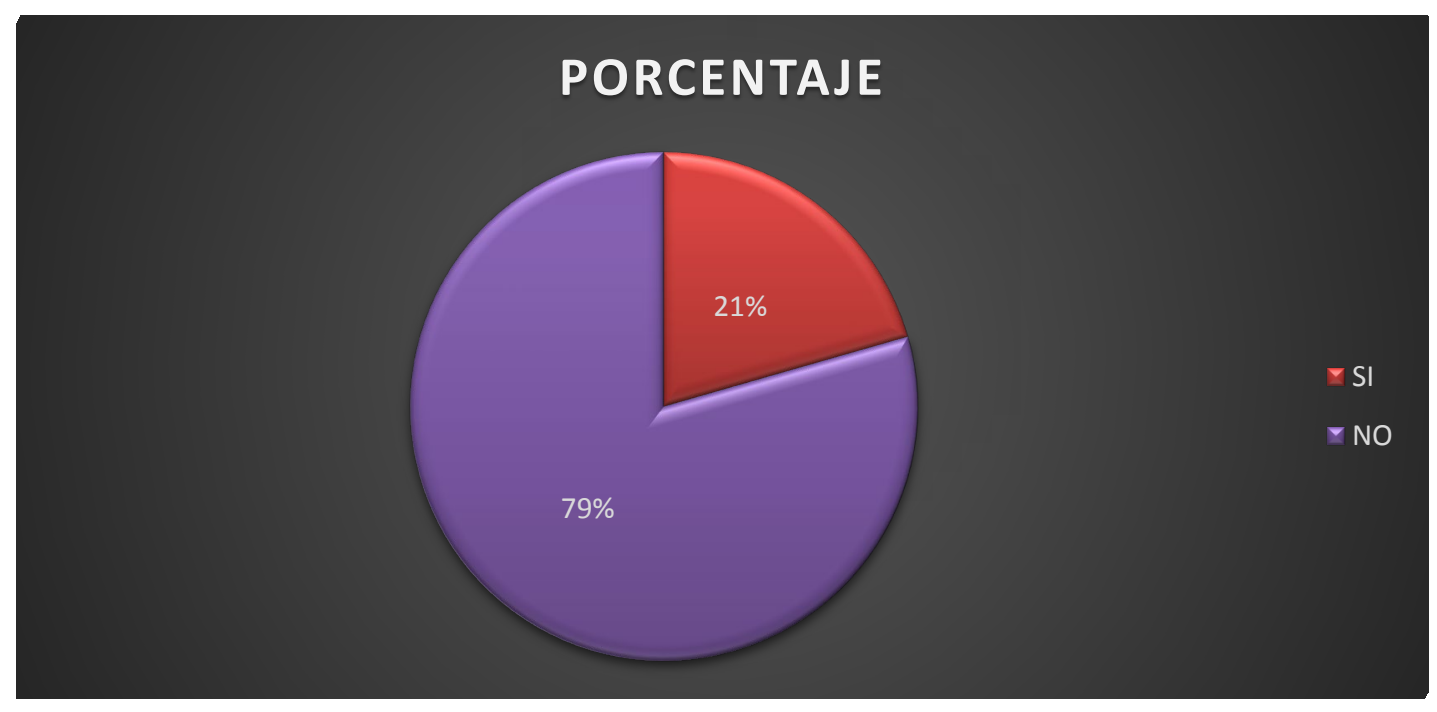

Gráfico 10 ¿Considera usted que dentro del proceso de enseñanza y aprendizaje se incluya cualquier tipo de religión para la solución de una problemática de intervención profesional?

Fuente: Estudiantes de los niveles cuarto "A", octavo "A" $\mathrm{y}$ "B" y noveno "A" de la facultad de Trabajo Social Elaborado por: Katherine Monserrate Cevallos Alcívar 
Interpretación.- Se puede observar que con un porcentaje del 79,44\% el cual es considerable, los estudiantes de la carrera creen que no se debería incluir de ninguna manera la religión en el ámbito educativo, ya que esto no les ayudará a la hora de la intervención profesional; por otro lado con el $21 \%$ los estudiantes encuestados expresaron que ellos piensan que es importante que se hable o se incluya la religión en sus cátedras, ya que ellos consideran que esto les ayudará a dar solución a las problemáticas que se les presenten a lo largo de su carrera profesional.

Respecto a tus creencias religiosas y posturas frente a la religión ¿Cuán importante es para ti que la gente conozca tus creencias religiosas?

Tabla 11 Respecto a tus creencias religiosas y posturas frente a la religión ¿Cuán importante es para ti que la gente conozca tus creencias religiosas?

\begin{tabular}{|l|l|l|}
\hline OPCIÓN & TOTAL DE RESPUESTAS & PORCENTAJE \\
\hline MUY IMPORTANTE & 10 & $9,35 \%$ \\
\hline IMPORTANTE & 31 & $28,97 \%$ \\
\hline POCO IMPORTANTE & 66 & $61,68 \%$ \\
\hline TOTAL & 107 & $100,00 \%$ \\
\hline
\end{tabular}

Fuente: Estudiantes de los niveles cuarto "A", octavo "A" y "B" y noveno "A" de la facultad de Trabajo Social 
ELABORADO POR: Katherine Monserrate Cevallos Alcívar

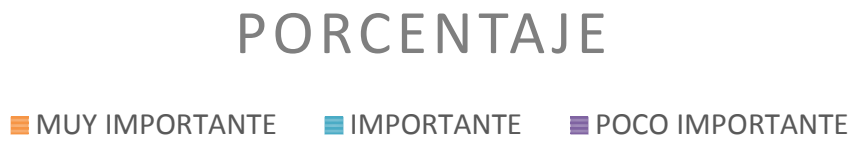

$70,00 \%$

\section{$60,00 \%$}

$50,00 \%$

$40,00 \%$

$30,00 \%$

$20,00 \%$

$10,00 \%$

$0,00 \%$

$9,35 \%$

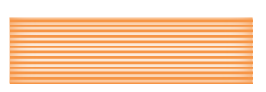

$28,97 \%$

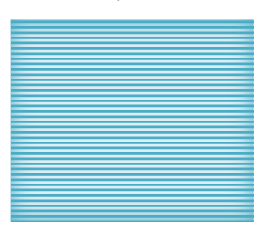

$61,68 \%$

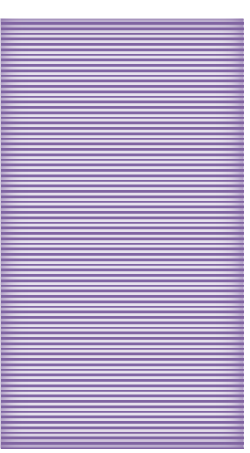

FUENTE:

Estudiantes de los niveles cuarto "A", octavo "A" y "B" y noveno "A" de la facultad de Trabajo Social

Elaborado por: Katherine Monserrate Cevallos Alcívar

Interpretación. - Se puede evidenciar que con el 61,68\% de la población estudiantil encuestada consideran que para ellos es poco importante que la gente conozca sus creencias religiosas, mientras que con el 28,97\% manifestaron que es importante que se conozca su religión y todo lo que respecta a ella, y finalmente con el 9,35\% los estudiantes creen que es muy importante que las personas conozcan su religión y sus creencias religiosas.

\section{¿Cuál es la postura de tu familia respecto a la religión?}

Tabla 12 ¿Cuál es la postura de tu familia respecto a la religión?

\begin{tabular}{|l|l|l|}
\hline OPCIÓN & TOTAL DE RESPUESTAS & PORCENTAJE \\
\hline COMPROMETIDO CON LA RELIGIÓN & 72 & $67,29 \%$ \\
\hline INDIFERENTE A LA RELIGIÓN & 32 & $29,91 \%$ \\
\hline CONTRARIA A LA RELIGIÓN & 3 & $2,80 \%$ \\
\hline TOTAL & 107 & $100,00 \%$ \\
\hline
\end{tabular}

Fuente: Estudiantes de los niveles cuarto "A", octavo "A" y "B" y noveno "A" de la facultad de Trabajo Social

ELABORADO POR: Katherine Monserrate Cevallos Alcívar

\footnotetext{
\begin{tabular}{l|l}
311 & $\begin{array}{l}\text { Vol. 5, núm. 3, julio, 2019, pp.293-316 } \\
\text { Patricia Josefa López Mero, Katherine Monserrate Cevallos Alcívar }\end{array}$
\end{tabular}
} 


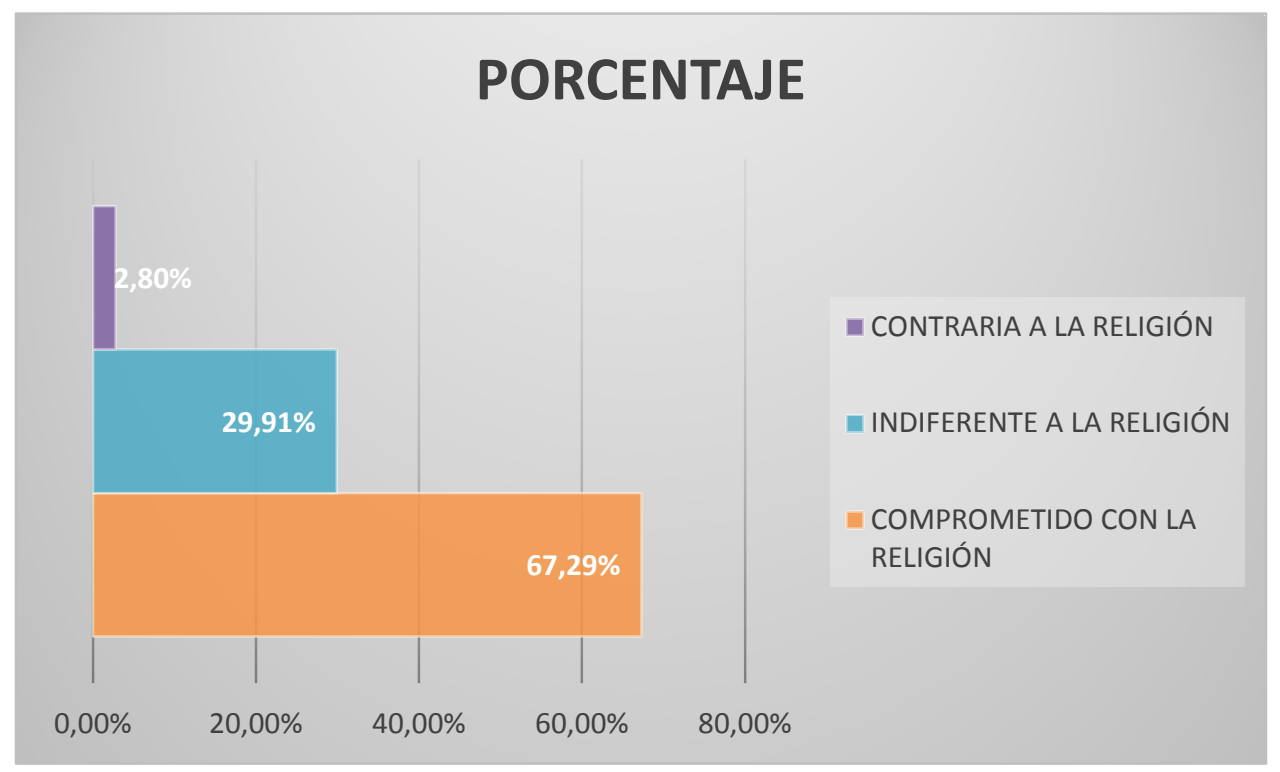

Gráfico 11 ¿Cuál es la postura de tu familia respecto a la religión?

FUENTE: Estudiantes de los niveles cuarto "A", octavo "A" y "B" y noveno "A" de la facultad de Trabajo Social ELABORADO POR: Katherine Monserrate Cevallos Alcívar

Interpretación. - A través de cuadro estadístico se puede observar que el 67,29 de los estudiantes consideran que su familia es comprometida con la religión; mientras que el 29,91\% manifestaron que en lo que respecta a sus familias ellos eran indiferentes a la religión y finalmente con un porcentaje mínimo como es el 2,80\% las familias de los estudiantes son contrarias a la religión.

\section{¿Considera usted que la religión contribuye en su formación profesional?}

Tabla 13 ¿Considera usted que la religión contribuye en su formación profesional?

\begin{tabular}{|l|l|l|}
\hline OPCIÓN & TOTAL DE RESPUESTAS & PORCENTAJE \\
\hline SI & 26 & $24,30 \%$ \\
\hline NO & 81 & $75,70 \%$ \\
\hline TOTAL & 107 & $100,00 \%$ \\
\hline
\end{tabular}

Fuente: Estudiantes de los niveles cuarto "A", octavo "A" y "B" y noveno "A" de la facultad de Trabajo Social

Elaborado por: Katherine Monserrate Cevallos Alcívar 


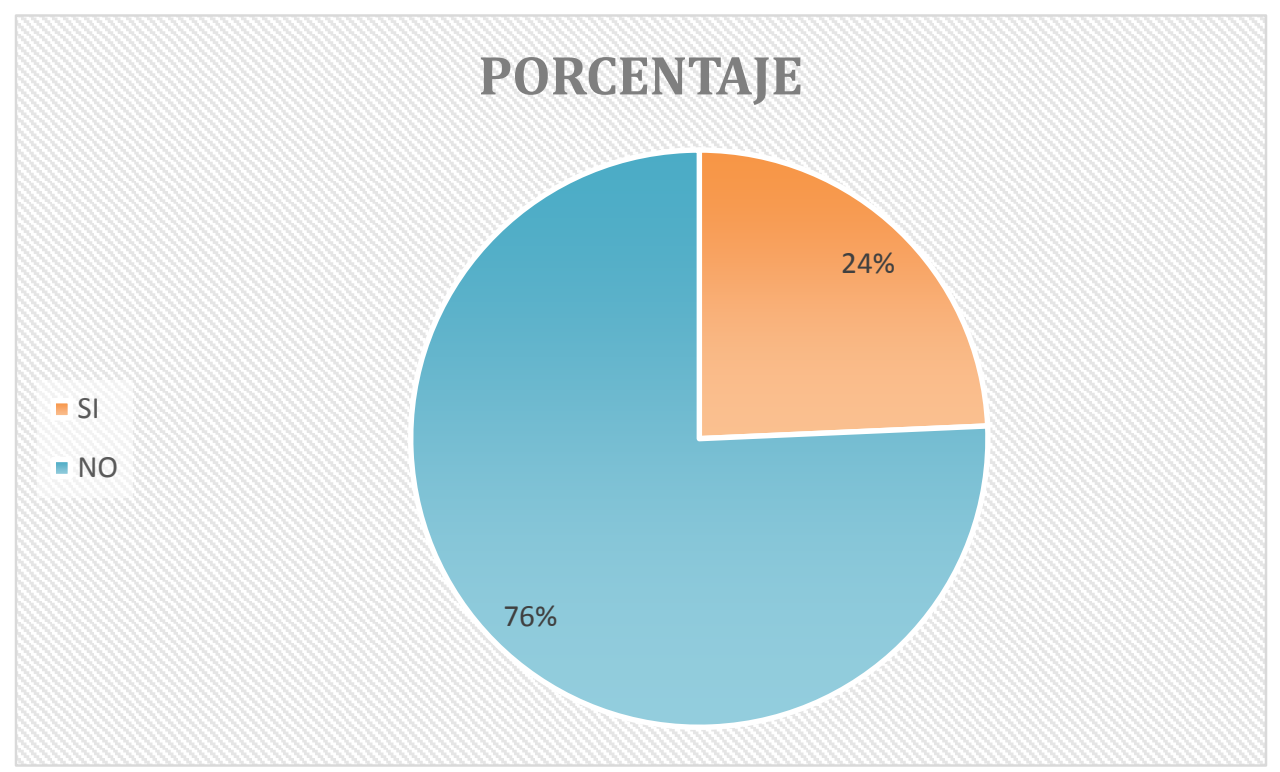

Gráfico 12 ¿Considera usted que la religión contribuye en su formación profesional?

FUENTE: Estudiantes de los niveles cuarto "A", octavo "A" y "B" y noveno "A" de la facultad de Trabajo Social ELABORADO POR: Katherine Monserrate Cevallos Alcívar

Interpretación.- Se puede observar a través del cuadro estadístico que el 76\% de los estudiantes que fueron encuestados consideran que la religión no les contribuye en su formación profesional, ya que dentro de la carrera se utilizan diversas teorías y además se usan metodologías, métodos, instrumentos, técnicas y modelos que ayudan en la intervención de las problemáticas sociales que respectan al Trabajador Social, por otro lado, con un mínimo porcentaje del $24 \%$ los estudiantes manifestaron que para ellos la religión es la base fundamental, no solo para la carrera sino a lo largo de su vida.

La hipótesis planteada fue: La religión de los estudiantes de la carrera de Trabajo Social incide negativamente en la formación profesional-ULEAM 2018. Esta hipótesis fue analizada y revisada en las preguntas número 13 y 2 de la encuesta que se le realizó a los estudiantes de los niveles cuarto "A" octavo "A" -"B" y noveno "A" de la Facultad de Trabajo Social de la universidad Laica "Eloy Alfaro" de Manabí, de tal manera que se llegó a la conclusión: que la religión no incide de manera negativa en su formación profesional, ya que de acuerdo a las respuestas de los estudiantes 
se puede evidenciar que en los ámbitos que más influye la religión es el familiar y con un pequeño porcentaje el educativo.

\section{Conclusiones}

- Los estudiantes de cuarto "A", octavo "A"-“B”, noveno "A" de la Facultad de Trabajo Social profesan las siguientes religiones: El 77,57\% es Católico; el 7,48\% Cristiano; el 2,80\% Evangélico; el 2,80\% Ateo; el 1,87\% mormón y el 7,48\% otras religiones;

- Los estudiantes encuestados consideran que la religión influye en los ámbitos: Familiar el 52\%, Personal 31\%, Social 8\%, otros ámbitos el 7\% y Educativo 2\%.

- $\quad$ Para el 76\% de los estudiantes encuestados de la Facultad de Trabajo Social, la religión no influye en su formación profesional porque cada docente se rige por los contenidos de las asignaturas que refleja el mesocurriculo y Rediseño Curricular de la carrera; pero también se evidenció que $21 \%$ de estudiantes consideran que a veces es necesario incluir la religión en los procesos de enseñanza aprendizaje para la solución de una problemática de intervención profesional.

\section{Recomendaciones}

- Los estudiantes y docentes deben mostrar respeto y aceptación por la diversidad de religión que profesan cada uno.

- Los temas relacionados a la religión deben ser considerados en los ámbitos Familiar, Personal, Social y en la educación no en todos los niveles debido a que, por la diversidad, no pueden ser aceptados.

- $\quad$ En el proceso de enseñanza-aprendizaje no se recomienda la inclusión de la religión para la solución de una problemática social además de que, a la hora de elegir una carrera, sea por vocación y convicción y no un espacio para dar a conocer temas de religión.

\section{Referencias Bibliográficas}

\footnotetext{
\begin{tabular}{|l|l}
314 & $\begin{array}{l}\text { Vol. 5, núm. 3, julio, 2019, pp.293-316 } \\
\text { Patricia Josefa López Mero, Katherine Monserrate Cevallos Alcívar }\end{array}$
\end{tabular}
} 
Consejo General de Trabajo Social. (2017). Consejo General de Trabajo Social. Obtenido de Consejo General de Trabajo Social: https://www.cgtrabajosocial.es/DefinicionTrabajoSocial

Constitución política del Ecuador. (2008). [Const.].

Enciclopedia de Clasificaciones. (2017). Enciclopedia de Clasificaciones. Obtenido de Tipos de. org: https://www.tiposde.org/sociedad/492-tipos-de-religion/

FEDFernández García, T., \& Ponce de León Romero, L. (2014). Nociones básicas de Trabajo Social. Madrid: Ediciones Académicas.

García, T. (2008). El concepto de religión en el sistema de la filosofía. España: Salamanca.

Garro, J. J. (2009). Modelos de intervención en Trabajo Social .

Gilligan, P., \& Furness, S. (2013). "Religión y espiritualidad en la formación y la práctica del trabajo social en el Reino Unido". Educación Social. Revista de Intervención Socioeducativa, $34 . \quad$ Obtenido de Google: https://www.raco.cat/index.php/EducacioSocial/article/viewFile/275580/368873

Lorda, L. M. (2013). El catolicismo en los orígenes del trabajo social: de la beneficencia y la caridad al Servicio Social. (Argentina, primera mitad del siglo XX. Revista de Trabajo Social - FCH - UNCPBA, 229.

Maria del Carmen Torres Alonso, \& Denny Cabrera Acosta. (2015). ACERCA DE LOS MODELOS DE INTERVENCIÓN EN TRABAJO SOCIAL. Obtenido de http://xn--caribea9za.eumed.net/wp-content/uploads/intervencion.pdf

Martínez, J. P. ( 2015). Especialización y límites en la práctica profesional relacionada con la dimensión religiosa espiritual. En J. P. Martínez, Espiritualidad y Trabajo Social controversias y oportunidades (págs. 277-278-279). Mexico: Universidad Autonoma Mejico. 
Palomino, R. (2009). Religión y Educación Pública. Universidad Comtuplense.

Philip Gilligan, S. F. (2014). Religión y espiritualidad en la formación y la practica de Trabao social en el Reino Unido. Educación Social. Revista de Intervención Socioeducativa, 33.

Ramírez, L. M. (2000). ACERCA DEL TRABAJO SOCIAL. Binasss.

Riquelme., S. F. (1. Enero 2017). La teoría en la Intervención social. Modelos y enfoques para el Trabajo social del siglo XXI. Revista coeditada por el IPS. Instituto de Política social y SocialMurcia, 25. 